\title{
Irreversible growth plate fusions in children with medulloblastoma treated with a targeted hedgehog pathway inhibitor
}

\author{
Giles W. Robinson ${ }^{1}$, Sue C. Kaste ${ }^{2}$, Wassim Chemaitilly ${ }^{3}$, Daniel C. Bowers ${ }^{4}$, Stephen \\ Laughton ${ }^{5}$, Amy Smith ${ }^{6}$, Nicholas G. Gottardo', Sonia Partap ${ }^{8}$, Anne Bendel ${ }^{9}$, Karen \\ D. Wright ${ }^{10}$, Brent A. Orr ${ }^{11}$, William C. Warner ${ }^{12}$, Arzu Onar-Thomas ${ }^{13}$ and Amar \\ Gajjar $^{1}$ \\ ${ }^{1}$ Department of Oncology, Division of Neuro-Oncology, St. Jude Children's Research Hospital, Memphis, TN, USA \\ ${ }^{2}$ Department of Radiological Sciences, Division of Diagnostic Imaging, St. Jude Children's Research Hospital, Memphis, TN, \\ USA \\ ${ }^{3}$ Department of Pediatric Medicine, Division of Endocrinology, St. Jude Children's Research Hospital, Memphis, TN, USA \\ ${ }^{4}$ Division of Pediatric Hematology and Oncology, University of Texas Southwestern Medical Center, Dallas, TX, USA \\ ${ }^{5}$ Starship Blood and Cancer Centre, Starship Children's Hospital, Auckland, NZ, USA \\ ${ }^{6}$ Division of Pediatric Hematology/Oncology, The Haley Center for Children's Cancer and Blood Disorders at Arnold Palmer \\ Hospital, Orlando, FL, USA \\ 7 Department of Haematology and Oncology, Princess Margaret Hospital for Children, School of Paediatrics and Child Health, \\ Telethon Kids Cancer Centre, Telethon Kids Institute, University of Western Australia, Perth, Western Australia, Australia \\ 8 Department of Neurology, Division of Child Neurology, Lucile Packard Children's Hospital at Stanford, Stanford University, \\ Palo Alto, CA, USA \\ ${ }^{9}$ Cancer and Blood Disorders Program, Children's Hospitals and Clinics of Minnesota, Minneapolis, MN, USA \\ 10 Pediatric Medical Neuro-Oncology, Dana-Farber Boston Children's Cancer and Blood Disorders Center, Harvard Medical \\ School, Boston, MA, USA \\ 11 Department of Pathology, St. Jude Children's Research Hospital, Memphis, TN, USA \\ 12 Department of Orthopedic Surgery, Campbell Clinic, University of Tennessee College of Medicine, Germantown, TN, USA \\ ${ }^{13}$ Department of Biostatistics, St. Jude Children's Research Hospital, Memphis, TN, USA \\ Correspondence to: Giles W. Robinson, email: giles.robinson@stjude.org \\ Keywords: premature physeal fusion, medulloblastoma, hedgehog inhibitor, targeted therapy, childhood toxicity \\ Received: August 16, $2017 \quad$ Accepted: August 21, $2017 \quad$ Published: September 01, 2017
}

Copyright: Robinson et al. This is an open-access article distributed under the terms of the Creative Commons Attribution License 3.0 (CC BY 3.0), which permits unrestricted use, distribution, and reproduction in any medium, provided the original author and source are credited.

\section{ABSTRACT}

The permanent defects in bone growth observed in preclinical studies of hedgehog (Hh) pathway inhibitors were not substantiated in early phase clinical studies of vismodegib in children. Consequently, vismodegib advanced into pediatric trials for malignancies suspected of being driven by aberrant activation of the Hh pathway. In one multicenter phase II trial, vismodegib was added to the therapy regimen for newly diagnosed Hh pathway activated medulloblastoma. Herein, we report on 3 children ( 2 on trial and one off trial) treated with vismodegib who developed widespread growth plate fusions that persist long after cessation of therapy. Currently, all 3 patients exhibit profound short stature and disproportionate growth, and 2 subsequently developed precocious puberty. Notably, the growth plate fusions only developed after a prolonged exposure to the drug ( $>140$ days). These findings resulted in a major trial amendment to restrict the agent to skeletally mature patients as well as a product label warning and update. Moreover, these findings alter the risk-benefit ratio of Hh inhibitors and underscore the importance of careful study of targeted agents in children. 


\section{INTRODUCTION}

Medulloblastoma is the most common malignant brain tumor in children. Standard therapy consists of surgery in combination with craniospinal irradiation (CSI) and chemotherapy. Although this treatment cures $60 \%$ $70 \%$, survivors of childhood medulloblastoma, experience significant life-long treatment-related morbidities. [1] Neurocognitive impairment, endocrinopathies, and early mortality occur as a consequence of the same broadbased cytotoxic modalities used to combat the disease. [2] Hence, therapy that preferentially targets diseased cells while sparing healthy tissue is eagerly anticipated.

Molecular studies of medulloblastoma show that approximately $25 \%$ are Hh pathway activated (hereafter designated as SHH-MB) and inhibitors targeting this pathway, such as vismodegib, sonidegib, and taladegib have been developed for clinical use. [3, 4] Preclinical studies showed these inhibitors were highly potent against mouse models of SHH-MB and had a low toxicity profile in adult mice; [5] however, studies in young mice uncovered severe and permanent defects in bone growth after short (2 day) exposures. [6]

Given these adverse effects in young mice, early phase clinical trials of vismodegib in children included serial monitoring for bone and dental toxicities. Thirtyone skeletally immature children received the Hh pathway inhibitor in phase I $(n=23)$ and phase II $(n=8)$ trials and none had bone or dental toxicities. [7, 8] Moreover, these studies demonstrated anti-tumor activity and prolonged progression-free survival in patients with relapsed $\mathrm{SHH}-$ MB. [8]

In 2013, St. Jude Children's Research Hospital initiated SJMB12 (NCT01878617), a multicenter phase II trial for newly diagnosed medulloblastoma that stratified patients to separate treatment arms by molecular and clinical risk. Vismodegib [dose range 114 - $225 \mathrm{mg}$ per body surface area $\left(\mathrm{m}^{2}\right)$ daily x 28 days x 12 cycles] was added after a slightly reduced standardized therapy regimen for SHH-MB patients to evaluate tolerability and study outcomes relative to historical cohorts. This marks the first time that a Hh pathway inhibitor has been used in the up-front treatment setting.

Here, we report 3 patients with SHH-MB treated with vismodegib who developed growth plate fusions. Of them, 2 patients were treated in SJMB12 and 1 patient received single-agent vismodegib off-study after disease relapse.

\section{PATIENT 1}

This 5-year-old white female was recently reported to have premature physeal closure after completing 5 cycles of vismodegib. [9] She was diagnosed with SHH-MB at 2 years of age and treated in a St. Jude institutional protocol aimed at reducing radiation exposure in young children with newly diagnosed brain tumors (NCT00602667). At disease relapse, the patient was taken off protocol therapy and the risks and benefits of additional treatment options such as salvage CSI, intravenous chemotherapy, and vismodegib were discussed with her family. Vismodegib therapy was chosen and informed consent obtained. Constrained by the pill strength of 150 $\mathrm{mg}$, the dose approximated $250 \mathrm{mg} / \mathrm{m}^{2}$. After 3 months of therapy, magnetic resonance imaging (MRI) showed a near-complete radiographic response and treatment was continued. Toward the end of cycle 5, she complained of bilateral lower extremity pain severe enough to wake her from sleep. A knee X-ray revealed incomplete centralized closure of bilateral proximal tibial and distal femoral physes, which was absent from findings of the knee X-ray taken 4 months earlier (Figure 1). MRI of the brain and spine revealed disease recurrence with leptomeningeal spread. Therefore, vismodegib therapy was discontinued.

Currently, 33 months after the second relapse, the patient continues to battle recurrent disease. Twice she entered remission while receiving cytotoxic chemotherapy, but relapsed within months of stopping therapy. Finally, after her fourth relapse, she underwent CSI and is currently 6 months from completion without evidence of disease.

Over time, effects of physeal fusions have become more evident. Although short stature may in part be attributable to prolonged therapy, her height, which was at the 43 rd percentile at 36 months of age, dropped to less than 3rd percentile by 60 months of age (Table 1). Bony protrusions have developed around her wrists, ankles, and knees. Her hands exhibit mild radial deviation around the wrist, with notable projection of the ulnar head. Her ankles demonstrate varus deformity, with the lateral malleolus appearing to overhang the normal position. Prominent fibular heads can be palpated around lateral aspects of her knees. Radiography of bones and joints show widespread systemic physeal fusions with increased metaphyseal sclerosis in phalanges of hands and feet, proximal humeri, distal radii, proximal and distal tibiae, femora, and fibulae (Figure 2). Despite this widespread distribution, all physes are not equally involved and presence of incomplete fusions, alongside complete ones, raise concern that disproportionate bone growth might eventually restrict her ability to walk and use hands normally.

Aberrations in Hh pathway genes PTCH1, PTCH2, and $S U F U$ are associated with nevoid basal cell carcinoma syndrome (NBCCS; also known as Gorlin syndrome), which predisposes patients to SHH-MB, basal cell carcinoma, and skeletal abnormalities. However, upon testing, this patient harbored no germline mutations in these genes.

\section{PATIENT 2}

An 8-year-old black female was diagnosed with SHH-MB at 5 years of age. Her medical history was 
remarkable for congenital bilateral radial and ulnar synostoses and a family history of medulloblastoma in both her father and paternal aunt. Fluorescence in situ hybridization (FISH) analysis revealed hemizygous loss of PTCH1 in tumor cells; however, a PTCH1 abnormality was not detected upon germline testing. Additional genetic testing to evaluate for other predispositions was offered but declined by the family. Nevertheless, because of high clinical suspicion for NBCCS and resulting heightened sensitivity to ionizing radiation, CSI was omitted from therapy. Consequently, she received SJMB12 protocol therapy with whole posterior fossa radiation instead of CSI, adjuvant chemotherapy, and 12 cycles of vismodegib $\left(188 \mathrm{mg} / \mathrm{m}^{2} /\right.$ dose $)$. After 6 cycles of vismodegib, MRI of knees showed normal and patent physes. However, after 12 cycles, she had central bony bridging across physes of tibiae, fibulae, and femora. Despite stopping therapy, subsequent imaging revealed progression of central to peripheral closure (Figure 1).

After 10 months, breast budding and pubertal hair growth were noted. Laboratory values confirmed the diagnosis of central precocious puberty (Table 1).

\section{Patient 1}

\section{Before Fusion}
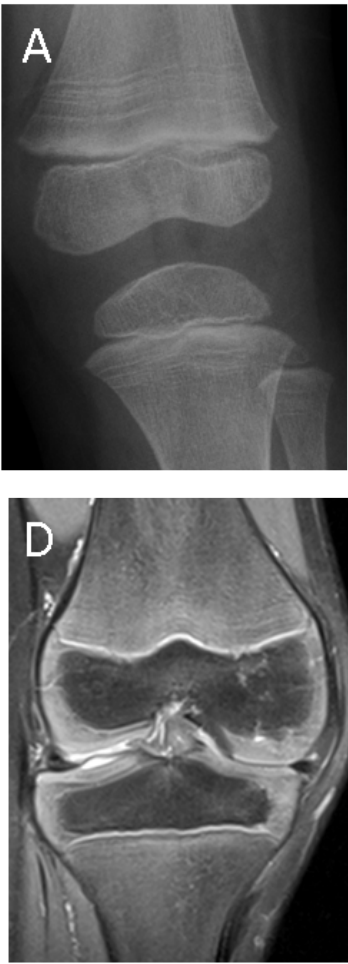

Patient 3

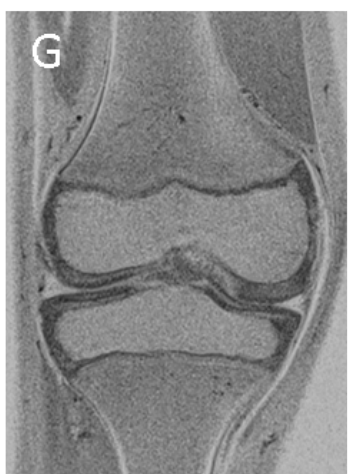

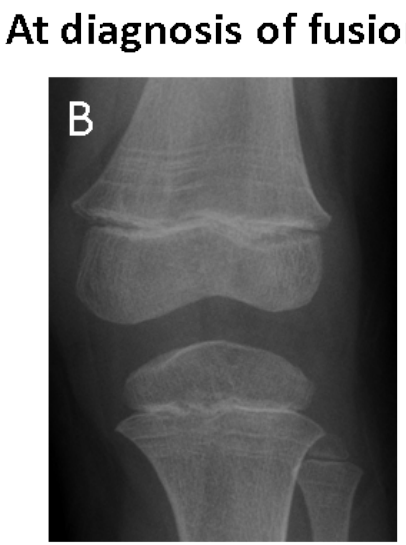
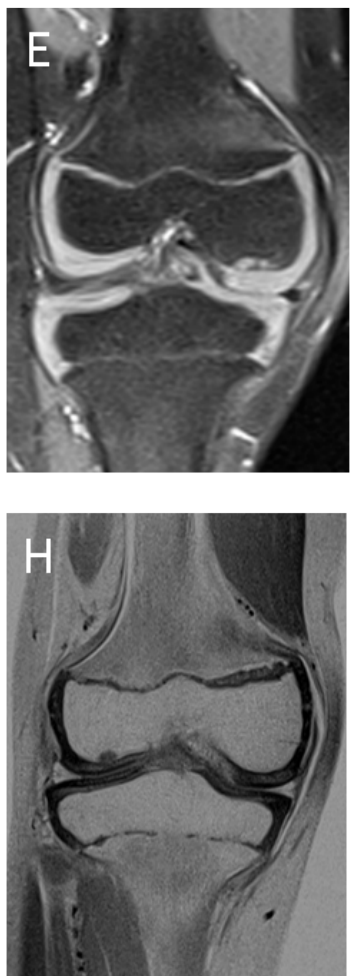

\section{At follow up}
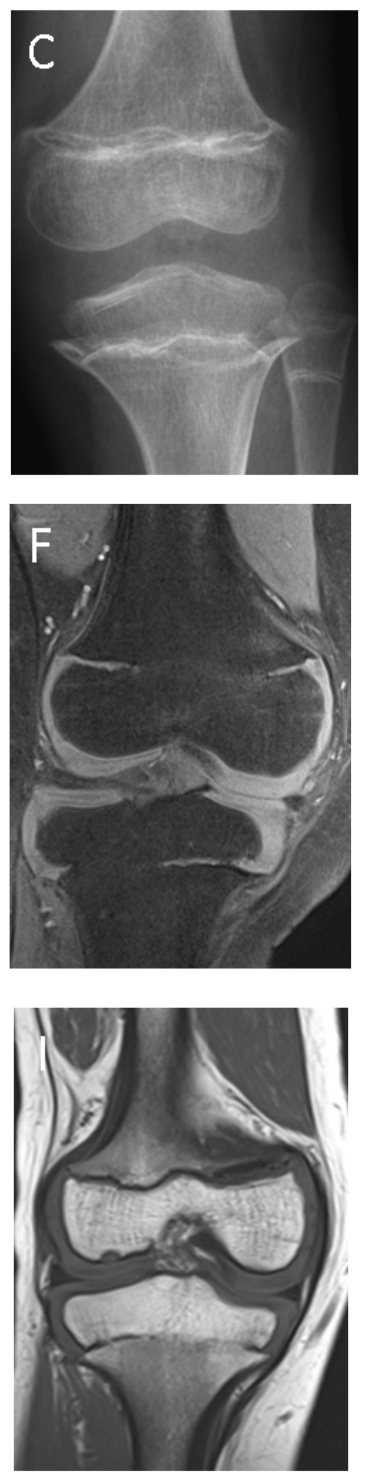

Figure 1: Imaging of knee in patients 1, 2, and 3 before fusion, at diagnosis of fusion, and at follow up. Baseline imaging (Panels A, D, and $\mathbf{G}$ ) show patent physes in all patients at the start of vismodegib therapy. In patient 1, physeal fusions were initially more pronounced in the proximal tibia, incomplete in the distal femur, and absent in the proximal fibula (Panel B). Follow-up radiography 17 months after stopping therapy (Panel $\mathbf{C}$ ) showed progression of fusions in all physes and development of abnormal metaphyseal sclerosis. In patients 2 and 3, MRI showed that the initial identification of fusion was subtle, with the development of small bridging fusions at the completion of 12 cycles of vismodegib therapy (Panels $\mathbf{E}$ and $\mathbf{H}$ ). Over time, these bridges widened to occupy more of the physeal stripe, as revealed by the MRI taken 6 months after the completion of vismodegib therapy (Panels $\mathbf{F}$ and $\mathbf{I}$ ). 
Table 1: Patient characteristics at primary diagnosis, 1 year post diagnosis, development of fusion, endocrine visit for fusion, and most recent endocrine visit

\begin{tabular}{|c|c|c|c|}
\hline Characteristic & Patient 1 & Patient 2 & Patient 3 \\
\hline Gender & $\mathrm{F}$ & $\mathrm{F}$ & $\mathrm{M}$ \\
\hline \multicolumn{4}{|l|}{ Characteristics at primary diagnosis } \\
\hline Age $-y$ & 2.3 & 4.9 & 6.7 \\
\hline Pubertal status & Tanner stage 1 & Tanner stage 1 & Tanner stage 1 \\
\hline Standing height - SD (percentile) & $-0.1(45 \%)$ & $1.0(84 \%)$ & $0.5(71 \%)$ \\
\hline Sitting height $-S D$ & Missing & 0 & 0.5 \\
\hline Discrepancy in standing to sitting height & Missing & Yes & No \\
\hline \multicolumn{4}{|l|}{ Characteristics at 1 y post-diagnosis evaluation } \\
\hline Age at 1 y post-diagnosis evaluation & 3.3 & 5.8 & 7.5 \\
\hline Time on vismodegib therapy - mo (cycles) & $3(3)$ & $3(3)$ & $3(3)$ \\
\hline Pubertal status & Tanner stage 1 & Tanner stage 1 & Tanner stage 1 \\
\hline Standing height -SD & $-0.4(35 \%)$ & $0.2(58 \%)$ & $0(48 \%)$ \\
\hline Sitting height -SD & Missing & 0 & -0.7 \\
\hline Discrepancy in standing to sitting height & Missing & No & No \\
\hline \multicolumn{4}{|l|}{ Characteristics at development of fusion } \\
\hline Age at development of fusion & 3.5 & 6.6 & 8.2 \\
\hline Time on vismodegib therapy - mo (cycles) & $5(5)$ & $11(12)$ & $11(12)$ \\
\hline Pubertal status & Tanner stage 1 & Tanner stage 1 & Tanner stage 1 \\
\hline Standing height - SD (Percentile) & $-0.6(28 \%)$ & $-0.2(40 \%)$ & $-0.6(29 \%)$ \\
\hline \multicolumn{4}{|l|}{ Characteristics at $1^{\text {st }}$ endocrine visit for fusion } \\
\hline Age $-y$ & 5.1 & 7.3 & 8.2 \\
\hline Total time of vismodegib therapy - mo & 5 & 11 & 11 \\
\hline Time since discontinuation of vismodegib - mo & 19 & 10 & 0 \\
\hline Pubertal status & Tanner stage 1 & Tanner stage 2 & Tanner stage 1 \\
\hline Clinical CPP & No & Yes & No \\
\hline Standing height - SD (percentile) & $-1.8(3 \%)$ & $-0.7(23 \%)$ & $-0.6(29 \%)$ \\
\hline Sitting height - SD & -1.0 & -0.5 & -1.0 \\
\hline Discrepancy in standing to sitting height & Yes & No & No \\
\hline GH peak $-\mathrm{ng} / \mathrm{mL}(n>5)$ & $4.7^{*}$ (failed) & 8.6 (passed) & $12.9+($ passed) \\
\hline LH baseline $-\mathrm{IU} / \mathrm{L}$ & 0.1 & 1.88 & 0.91 \\
\hline Estradiol baseline - pg/mL & 0 & 14 & Not applicable \\
\hline Testosterone - ng/dL & Not applicable & Not applicable & 0 \\
\hline LH stimulated - IU/L & Not assessed & Not assessed & $12.72+$ \\
\hline Testosterone, stimulated - ng/dL & Not assessed & Not applicable & $47 \dagger$ \\
\hline Laboratory results indicating central precocious puberty & No & Yes & Yest \\
\hline Bone age $-y$ & 5.0 & 13.5 & 12.5 \\
\hline Osseous abnormalities in bone age & Yes & Yes & Yes \\
\hline Treatment & None & $\mathrm{GnRHa}$ & $\mathrm{GnRHa}$ \\
\hline \multicolumn{4}{|l|}{ Characteristics at most recent endocrine visit } \\
\hline Age $-y$ & 5.8 & 8.8 & 9.8 \\
\hline Time since discontinuation of vismodegib - mo & 28 & 28 & 19 \\
\hline Pubertal status & Tanner stage 1 & Tanner stage 1 & Tanner stage 1 \\
\hline Clinical CPP & Not present & Suppressed & Suppressed \\
\hline Standing height - SD (percentile) & $-2.7(0 \%)$ & $-1.4(7 \%)$ & $-1.3(8 \%)$ \\
\hline Sitting height - SD & -2.0 & -1.0 & Missing \\
\hline Discrepancy in standing to sitting height & No & No & Missing \\
\hline Estradiol - pg/ mL & 0 & 0 & Not applicable \\
\hline $\mathrm{GH}$ peak - ng/mL $(n>5)$ & Not assessed & $9.6 \mathrm{ng} / \mathrm{mL}$ (Passed) & $4.5 \mathrm{ng} / \mathrm{mL}$ (failed) \\
\hline Testosterone - ng/dL & Not applicable & Not applicable & 0 \\
\hline Bone age $-y$ & Not assessed & 14.5 & 12.5 \\
\hline Osseous abnormalities in bone age & Not assessed & Yes & Yes \\
\hline Treatment & None & $\mathrm{GnRHa}$ & $\mathrm{GnRHa}$ \\
\hline Counseling & $\begin{array}{l}\text { Consider GH } \\
\text { replacement }\end{array}$ & & $\begin{array}{l}\text { Consider GH } \\
\text { replacement. }\end{array}$ \\
\hline
\end{tabular}

*GH testing done 6 months later.

$\uparrow$ Patient 3 underwent dynamic testing 3 months after initial changes in bone age were observed.

F, female; M, male; y, year; mo, month; SD, standard deviation; CPP, central precocious puberty; GH, growth hormone, LH, luteinizing hormone; GnRHa, gonadotropin- releasing hormone agonist. 
Gonadotropin releasing hormone agonist (GnRHa) was given for pubertal suppression to avoid further acceleration of physeal fusions.

Two years after stopping vismodegib, her height has gone from the $83 \mathrm{rd}$ percentile at 5 years to the 8 th percentile at 8 years. In addition, her bone age has advanced 6 years beyond her chronologic age to 14 years (Table 1 and Figure 2).

\section{PATIENT 3}

A 9-year-old white male was diagnosed at age 6 with desmoplastic/nodular variant medulloblastoma of the $\mathrm{SHH}$ subgroup. FISH analysis revealed hemizygous loss of PTCH1 in tumor cells. He was enrolled in SJMB12 and received 12 cycles of vismodegib $\left(167 \mathrm{mg} / \mathrm{m}^{2} / \mathrm{dose}\right)$. Screening MRIs of the right knee at start and after 6 cycles of vismodegib revealed normal patent physes. MRI of the same knee after 12 cycles showed early central fusion of the proximal tibial physis. Within 6 months, this physis became progressively and abnormally fused, predominantly in central and medial portions (Figure 1). A partially fused proximal femur physis was also observed with an advanced bone age of 12.5 years.

Although there were no signs of puberty, leuprolide stimulation testing performed 3 months after the bony fusion was consistent with pubertal response, indicating imminent onset of central puberty. Pubertal suppression with GnRHa was initiated for this patient.

Currently, 19 months after stopping vismodegib, his height has gone from the 70th percentile at 6 years to less than the 10th percentile at 9 years (Table 1). Skeletal survey revealed widespread partial and complete physeal fusions similar to those in patient 1 (Figure 2).

\section{DISCUSSION}

The Hh pathway promotes growth, symmetry, and differentiation in various tissues. [10] In mammals, this pathway is activated by the ligands sonic hedgehog (SHH), Indian hedgehog ( $\mathrm{IHH})$, and desert hedgehog $(\mathrm{DHH})$. These ligands are specifically expressed in distinct compartments and conservation of these is a mechanism by which developing bodies mediate growth of different tissue types through a single intracellular pathway. [11] $\mathrm{SHH}$ primarily activates granule neural cell precursor proliferation in the developing cerebellum, IHH drives chondrocyte proliferation in growth plates of the bone, and DHH is active almost exclusively in gonads. [11] All ligands bind the transmembrane receptor Patched 1 (PTCH1) which triggers its removal from the cilium. This removal of PTCH1 allows the protein smoothened (SMO) to translocate into the cilium where, upon activation, it triggers a signal cascade within the cell. $[12,13]$

Current $\mathrm{Hh}$ pathway inhibitors function by suppressing SMO, regardless of the Hh ligand. In adults, probably owing to the diminished role of $\mathrm{Hh}$ signaling, these agents cause muscle spasms, alopecia, dysgeusia, fatigue, and weight loss. [14] In children, given the pervasiveness of this pathway in developing organ systems, these inhibitors could theoretically have farreaching systemic consequences.

Indeed, here we describe 3 cases of growth plate fusions in patients on vismodegib and a very recent publication also describes 3 additional cases seen in patients receiving the $\mathrm{Hh}$ inhibitor, sonidegib (LDE225). [15] These 6 cases clearly show that disruption of the Hh pathway through SMO antagonism can affect developing humans in a similar manner as developing mice. In mice, exposure to SMO inhibitor resulted in blocked proliferation and early differentiation of chondrocytes, followed by a marked increase in mineralization within the growth plate once the agent was withdrawn; [6] a process that bears eerie resemblance to what has been described in these 3 cases. For the mice, the end result was the shortening of bones and dysplastic joint formation that drew parallels to signs associated with IHH deficiency. [6] Intriguingly, some of the growth abnormalities observed, particularly in Patients 1 and 3 (Figure 2), are also akin to those in patients born with IHH deficiency, which causes the autosomal recessive syndrome acrocapitofemoral dysplasia that is characterized by short-limb dwarfism and brachydactyly. [16]

But how common is this toxicity? Toxic effects related to $\mathrm{Hh}$ inhibitors in just 6 patients might not seem substantial given that many children receiving SMO inhibitors have not presented with bone toxicity; however, when duration of exposure and the small number of skeletally immature patients treated are factored in, this number becomes concerning. One important distinction between mouse and man is that bone growth in mice is by comparison extremely rapid and complete at 21 days old. Hence, owing to the much slower growth rate, the time-to-toxicity will predictably be much slower. Indeed the growth plate fusions described here were seen only in patients exposed to 5 or more cycles ( $>140$ days), whereas, in early phase clinical trials, median exposure to vismodegib was 2 cycles (56 days). [7] In the phase II study only 1 of 25 received more than 5 cycles of vismodegib and this patient progressed and withdrew from therapy in cycle 6 (162 days). [8] Similarly in the recently published phase I/II trial of sonidegib (LDE225) the 3 pediatric patients who developed growth plate abnormalities discontinued therapy at 57, 169, and 196 days, respectively. By comparison, the median treatment exposure for the pediatric population $(n=60)$ was 55 days suggesting that the vast majority failed to stay on the drug for more than 2 cycles. [15] Consequently, prolonged exposure appears necessary for development of growth deficits and likely explains the paucity of growth plate findings to date.

One major concern is that if the use of SMO 
inhibitors becomes more commonplace in pediatric oncology then the incidence of this toxicity will incrementally rise. As of February 2016 (when this toxicity was recognized), $23 \mathrm{SHH}-\mathrm{MB}$ patients were enrolled in SJMB12. Of them, 9 were skeletally mature (defined as females $\geq 15$ years old and males $\geq 17$ ). Of the remaining 14 patients, 8 did not receive vismodegib: 3 had not reached this point in therapy, 1 could not swallow tablets, 2 progressed before vismodegib administration, and 2 opted out of study. Of 6 remaining patients, 2 progressed before completing 3 cycles and 4 remained on vismodegib for 5 or more cycles. Two of the 4 (Patient
\#2 and \#3) who completed 12 cycles developed physeal fusion. The other 2 patients, a $9 \mathrm{yr}$ female and a $10 \mathrm{yr}$ old male, completed 6 and 7 cycles, respectively, and had no radiographic evidence of fusion 10 and 17 months from starting vismodegib therapy. Therefore, the actual incidence of this adverse event is currently $50 \%$ for skeletally immature patients in SJMB12 who completed 6 cycles and $100 \%$ for those who completed 12 cycles of vismodegib.

Even though short stature and growth abnormalities are seen in the pediatric population treated with CSI and intensive adjuvant chemotherapy, the growth

\section{Patient 1}

\section{Patient 2}
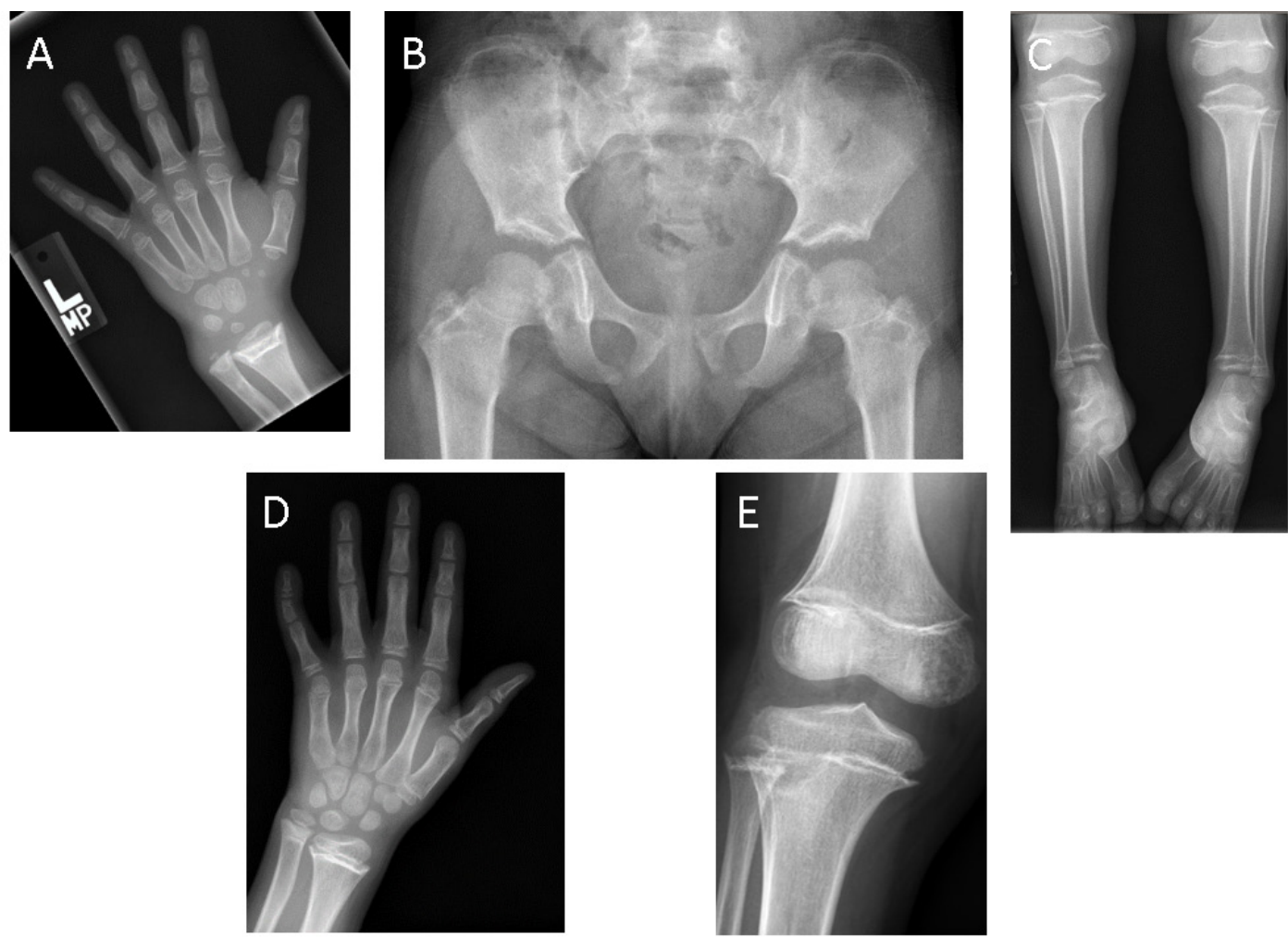

Patient 3
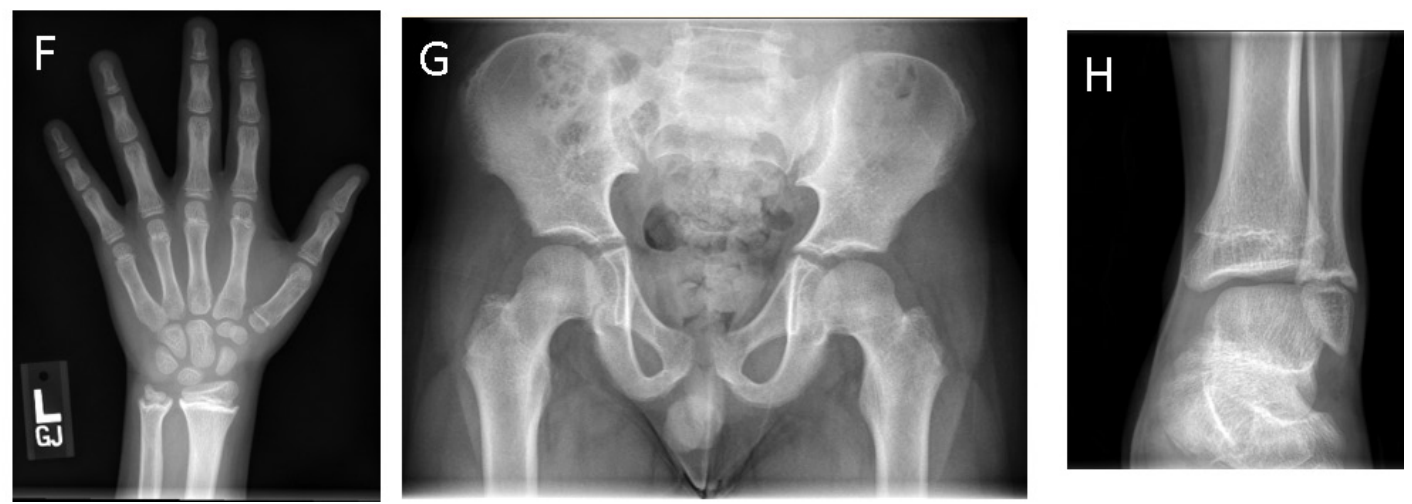

Figure 2: Imaging of patients at follow up showing skeletal deformities resulting from widespread physeal fusions. Radiographs of patient 1 at 18 months after cessation of therapy show protrusion of the ulnar head and widespread physeal fusion in hand bones (Panel A); abnormally short femoral necks (Panel B); and varus ankles, with widespread physeal fusions of bones in the lower leg (Panel C). Radiographs of patient 2 at 14 months after completion of vismodegib therapy show widespread physeal fusions in hand and forearm bones (Panel D) accompanied by sclerotic thickening in all physes of the knee (Panel E). Three radiographs of patient 3 taken 16 months after cessation of therapy show abnormalities that are less prominent but similar to those of patient 1 . Advanced bone age (Panel $\mathbf{F}$ ), shortened femoral necks (Panel $\mathbf{G}$ ), and distal fusion of the tibia with relative sparing of the fibula (Panel $\mathbf{H}$ ) was seen. 
abnormalities seen here are notably distinct. Patients receiving multimodality therapy may display short stature from treatment induced growth hormone $(\mathrm{GH})$ deficiency, spinal radiation generating poor sitting heights, and chemotherapy induced reduction in final height. [1719] However, premature physeal closure is not a finding associated with this multimodal therapy and patient \#2 developed a poor standing height despite not receiving CSI, not displaying GH deficiency, and not having a discrepancy in sitting and standing height.

The fact that 2 of the 3 patients with physeal fusion also developed precocious puberty is unexpected. Epiphyseal maturation occurs throughout puberty and is driven by a complex, incompletely understood signaling network involving $\mathrm{IHH}$, parathyroid hormonerelated protein, and bone morphogenetic proteins. [20] Consequently, it is possible that a feedback signal from bone to the hypothalamic-pituitary axis was altered due to premature cessation of Hh signaling. Although most interesting, the link between precocious puberty and these growth plate fusions cannot be substantiated on 2 patients alone and it requires a lot more exploration. Nonetheless, since testing is uncomplicated, this observation should elicit surveillance of all patients who experience this toxicity.

Another concern for these patients is that once a growth signal is stimulated, through endogenous $\mathrm{GH}$ release or through $\mathrm{GH}$ replacement, it may accelerate abnormal growth in more severely affected areas rather than increase the final height. Two patients reported herein have since developed growth hormone $(\mathrm{GH})$ deficiency as expected after CSI exposure [17] and the decision to replace is being debated. Patients have been counseled, and to date none of them opted for GH replacement. Given the anticipated severity of skeletal abnormalities, orthopedics and psychological counsel is being given to all the patients and their families such that an informed decision can be reached.

In conclusion, our observations raise questions about the use of Hh inhibitors in skeletally immature patients. Although responses to Hh inhibitors have sometimes been dramatic, they have been short-lived and restricted to a small subset of SHH-MB patients with defects in the $\mathrm{Hh}$ pathway upstream of SMO. $[8,21]$ Also, responders do not harbor TP53 mutations, which represent a significant subset of SHH-MB with poor prognosis. Therefore, in SJMB12, for newly diagnosed patients with SHH-MB for whom predicted survival after multimodal therapy remains high [3], the putative benefit of $\mathrm{Hh}$ inhibitors was not felt to outweigh the risks. Consequently, the protocol was amended to restrict vismodegib to skeletally mature patients. Furthermore, Genentech has updated its product label and issued a warning letter regarding the risk of premature epiphyseal fusion. [22, 23]

In children with incurable medulloblastoma for whom treatment options are limited or absent, the decision to use Hh inhibitors is complex. Tumors should be molecularly vetted against the agent's mechanism of action to see if these will predictably respond, and, in our opinion, Hh inhibitors should only be used in developing children if the potential benefit is greater than the debilitating risks.

In a broader context, while the introduction of targeted agents heralds an exciting era in medicine, agents that target developmental signaling pathways should be thoroughly evaluated for potential development-specific toxicities. Since pharmaceutical companies and clinical trialists are, correctly, being encouraged to include children in a novel agent's developmental path, the importance of careful monitoring for toxicities, particularly in the growing and developing child, cannot be overstated.

\section{ACKNOWLEDGMENTS}

This work was supported by American Lebanese Syrian Associated Charities (ALSAC). The SJMB12 study (NCT01878617) is in supported, in part, by Genentech. We thank Vani Shanker PhD, for reviewing and editing the manuscript.

\section{CONFLICTS OF INTEREST}

The authors have no conflicts of interest to declare.

\section{Editorial note}

This paper has been accepted based in part on peerreview conducted by another journal and the authors' response and revisions as well as expedited peer-review in Oncotarget.

\section{REFERENCES}

1. Ostrom QT, de Blank PM, Kruchko C, Petersen CM, Liao P, Finlay JL, Stearns DS, Wolff JE, Wolinsky Y, Letterio JJ, Barnholtz-Sloan JS. Alex's Lemonade Stand Foundation Infant and Childhood Primary Brain and Central Nervous System Tumors Diagnosed in the United States in 20072011. Neuro Oncol. 2015; 16 Suppl 10:x1-x36. doi: 10.1093/neuonc/nou327.

2. Armstrong GT, Liu Q, Yasui Y, Huang S, Ness KK, Leisenring W, Hudson MM, Donaldson SS, King AA, Stovall M, Krull KR, Robison LL, Packer RJ. Long-term outcomes among adult survivors of childhood central nervous system malignancies in the Childhood Cancer Survivor Study. J Natl Cancer Inst. 2009; 101:946-58. doi: 10.1093/jnci/djp148.

3. Kool M, Korshunov A, Remke M, Jones DT, Schlanstein M, Northcott PA, Cho YJ, Koster J, Schouten-van Meeteren A, van Vuurden D, Clifford SC, Pietsch T, von Bueren $\mathrm{AO}$, et al. Molecular subgroups of medulloblastoma: 
an international meta-analysis of transcriptome, genetic aberrations, and clinical data of WNT, SHH, Group 3, and Group 4 medulloblastomas. Acta Neuropathol. 2012; 123:473-84. doi: 10.1007/s00401-012-0958-8.

4. Gajjar AJ, Robinson GW. Medulloblastoma-translating discoveries from the bench to the bedside. Nat Rev Clin Oncol. 2014; 11:714-22. doi: 10.1038/nrclinonc.2014.181.

5. Romer JT, Kimura H, Magdaleno S, Sasai K, Fuller C, Baines H, Connelly M, Stewart CF, Gould S, Rubin LL, Curran T. Suppression of the Shh pathway using a small molecule inhibitor eliminates medulloblastoma in Ptc1(+/-) p53(-/-) mice. Cancer Cell. 2004; 6:229-40. doi: 10.1016/j. ccr.2004.08.019.

6. Kimura H, Ng JM, Curran T. Transient inhibition of the Hedgehog pathway in young mice causes permanent defects in bone structure. Cancer Cell. 2008; 13:249-60. doi: 10.1016/j.ccr.2008.01.027.

7. Gajjar A, Stewart CF, Ellison DW, Kaste S, Kun LE, Packer RJ, Goldman S, Chintagumpala M, Wallace D, Takebe N, Boyett JM, Gilbertson RJ, Curran T. Phase I study of vismodegib in children with recurrent or refractory medulloblastoma: a pediatric brain tumor consortium study. Clin Cancer Res. 2013; 19:6305-12. doi: 10.1158/10780432.CCR-13-1425.

8. Robinson GW, Orr BA, Wu G, Gururangan S, Lin T, Qaddoumi I, Packer RJ, Goldman S, Prados MD, Desjardins A, Chintagumpala M, Takebe N, Kaste SC, et al. Vismodegib Exerts Targeted Efficacy Against Recurrent Sonic Hedgehog-Subgroup Medulloblastoma: Results From Phase II Pediatric Brain Tumor Consortium Studies PBTC025B and PBTC-032. J Clin Oncol. 2015. doi: 10.1200/ JCO.2014.60.1591.

9. Lucas JT Jr, Wright KD. Vismodegib and Physeal Closure in a Pediatric Patient. Pediatr Blood Cancer. 2016; 63:2058. doi: $10.1002 /$ pbc. 25941 .

10. Lum L, Beachy PA. The Hedgehog response network: sensors, switches, and routers. Science. 2004; 304:1755-9. doi: 10.1126/science. 1098020 .

11. Varjosalo M, Taipale J. Hedgehog: functions and mechanisms. Genes Dev. 2008; 22:2454-72. doi: 10.1101/ gad.1693608.

12. Pak E, Segal RA. Hedgehog Signal Transduction: Key Players, Oncogenic Drivers, and Cancer Therapy. Dev Cell. 2016; 38:333-44. doi: 10.1016/j.devcel.2016.07.026.

13. Bangs F, Anderson KV. Primary Cilia and Mammalian Hedgehog Signaling. Cold Spring Harb Perspect Biol. 2017; 9. doi: 10.1101/cshperspect.a028175.

14. Sekulic A, Migden MR, Oro AE, Dirix L, Lewis KD, Hainsworth JD, Solomon JA, Yoo S, Arron ST, Friedlander PA, Marmur E, Rudin CM, Chang AL, et al. Efficacy and safety of vismodegib in advanced basal-cell carcinoma. N Engl J Med. 2012; 366:2171-9. doi: 10.1056/ NEJMoa1113713.
15. Kieran MW, Chisholm J, Casanova M, Brandes AA, Aerts I, Bouffet E, Bailey S, Leary S, MacDonald TJ, Mechinaud F, Cohen KJ, Riccardi R, Mason W, et al. Phase I study of oral sonidegib (LDE225) in pediatric brain and solid tumors and a phase II study in children and adults with relapsed medulloblastoma. Neuro Oncol. 2017. doi: 10.1093/neuonc/ nox 109 .

16. Hellemans J, Coucke PJ, Giedion A, De Paepe A, Kramer P, Beemer F, Mortier GR. Homozygous mutations in IHH cause acrocapitofemoral dysplasia, an autosomal recessive disorder with cone-shaped epiphyses in hands and hips. Am J Hum Genet. 2003; 72:1040-6.

17. Laughton SJ, Merchant TE, Sklar CA, Kun LE, Fouladi M, Broniscer A, Morris EB, Sanders RP, Krasin MJ, Shelso J, Xiong Z, Wallace D, Gajjar A. Endocrine outcomes for children with embryonal brain tumors after risk-adapted craniospinal and conformal primary-site irradiation and high-dose chemotherapy with stem-cell rescue on the SJMB-96 trial. J Clin Oncol. 2008; 26:1112-8. doi: 10.1200/ JCO.2008.13.5293.

18. Xu W, Janss A, Moshang T. Adult height and adult sitting height in childhood medulloblastoma survivors. J Clin Endocrinol Metab. 2003; 88:4677-81. doi: 10.1210/jc.2003030619.

19. Bruzzi P, Predieri B, Corrias A, Marsciani A, Street ME, Rossidivita A, Paolucci P, Iughetti L. Final height and body mass index in adult survivors of childhood acute lymphoblastic leukemia treated without cranial radiotherapy: a retrospective longitudinal multicenter Italian study. BMC Pediatr. 2014; 14:236. doi: 10.1186/14712431-14-236.

20. Kronenberg HM. Developmental regulation of the growth plate. Nature. 2003; 423:332-6. doi: 10.1038/nature01657.

21. Rudin CM, Hann CL, Laterra J, Yauch RL, Callahan CA, Fu L, Holcomb T, Stinson J, Gould SE, Coleman B, LoRusso PM, Von Hoff DD, de Sauvage FJ, et al. Treatment of medulloblastoma with hedgehog pathway inhibitor GDC-0449. N Engl J Med. 2009; 361:1173-8. doi: 10.1056/NEJMoa0902903.

22. Erivedge Prescribing Information. (South San Francisco, CA: Genentech USA Inc.). 2016.

23. Important Drug Warning Letter. (South San Francisco, CA: Genentech USA Inc.). 2016. 